\title{
Full resummation analysis of jet quenching and tests of the QCD Equation of State
}

\author{
X. Feal ${ }^{a, *}$ \\ ${ }^{a}$ Physics Department, Brookhaven National Laboratory \\ Bldg. 510A, Upton, NY 11973, USA \\ E-mail: xgarciafe@bnl.gov
}

Jet quenching has become a fundamental tool to study the hot QCD matter produced in heavy ion collisions. While important theoretical and experimental advances have been made in the last two decades, the extraction of the medium properties and the comparison with finite temperature QCD is still particularly worrisome. In this work we show that improvements in the calculation of the medium-induced gluon spectrum are required for a correct extraction of the parameters without temperature issues. In particular, we employ an improved numerical implementation of multiple hard scatterings that resums all terms in the opacity expansion beyond the Gaussian approximation. We find significant differences in the extracted medium parameters when comparing with two of the most used approximations in phenomenological analyses to date, the first order opacity expansion and the Gaussian approximation. We also make a first attempt to compare the extracted medium parameters with lattice results.

HardProbes 2020

1-6 June 2020

Austin, Texas

\footnotetext{
${ }^{*}$ Speaker
} 
The study of the properties of the hot QCD matter constitutes one of the main goals of the heavy ion program at LHC and RHIC [1-3]. A fundamental tool in these studies is provided by jet quenching observables. After a typical hard event a parton is expected to undergo multiple interactions with the hot and dense medium created in the collision of two heavy ions. Measuring the imprints of these interactions in the final spectrum of these particles provides, then, a natural way to explore the properties of the Quark Gluon Plasma (QGP).

Despite the current wealth of data and the large progress on the theoretical side over the last decades, the extraction of the QGP parameters through jet quenching analyses has been subject to some uncertainties. Current calculations for one of the simplest observables, the nuclear modification factor $R_{A A}^{h}$, which measures the depletion of high $p_{\perp}$ hadrons in the QGP, have been in goo agreement with the data for a given energy, centrality and colliding system. However, a common understanding of the RHIC and LHC data without temperature issues is still missing. Particularly, an inconsistent deviation in the extracted medium opacity has been reported by all the phenomenological analyses to date [4-6], making the QGP produced at RHIC look apparently $K \simeq 1.3-2$ times more dense than expected assuming the LHC temperature scenario. To this extent, it is natural to wonder whether these discrepancies can be attributed or not to the required approximations employed so far in the required calculations to predict the jet quenching phenomena.

The main contribution to jet quenching is the energy-loss of fast partons in the QGP through multiple gluon emission [7]. In a pQCD scenario, partons collide multiple times with QGP constituents, quantum mechanically rotating its color and momentum and giving rise thus to radiative corrections depleting the high energy states before hadronization. The resummation of this multiple scattering effects in the gluon intensity has been challenging, and the form of the spectrum after a hard collision vertex has been known for a while only in some approximations. In the single hard approximation $[8,9]$ only the first or first terms in an expansion in the number of collisions are kept. While this approximation provides a satisfactory description of the perturbative cross sections, it does not fully account the whole multiple scattering in the QGP, lacking then some sensitivity of the emission to the decoherence phenomena occurring in the soft gluon limit [10-12]. On the other hand, in the multiple soft - or Gaussian - approximation [13-18] arbitrary large opacities can be handled by relaxing the parton medium cross sections to Gaussian distributions. While this approximation seems good for the account of multiple soft scatterings, it misses the typically large tails $1 / q^{4}$ of the real cross sections, signaling collisions with point-like scatterers in the plasma, and thus lead to a substantial underestimation of the large $1 / \omega$ tails of the gluon spectrum.

These caveats and the issues in the extraction of the QGP parameters have recently triggered more precise calculations of the medium induced gluon radiation [19-25]. We propose here to determine if the absence of a full resummation of the multiple hard scattering has been consistently re-absorbed as a systematic error in the extracted parameters. To that end, we compare the extracted QGP opacity using either the first order perturbative result or including all the neglected terms in this expansion. We are able to show that this improvement is indeed required for a correct extraction of the QGP parameters without temperature issues. By the same token, we will be able to compare our results with the lattice predictions, opening up an additional handle to study the QCD Equation of State.

The intensity of soft gluons emitted in an in-medium path $l$ with energy between $\omega$ and $\omega+d \omega$, 
per unit of time and per unit of medium transverse size, up to all orders in the opacity, is given by

$$
\omega \frac{d I}{d \omega}=\alpha_{s} C_{R} \int \frac{d \Omega_{n}}{(2 \pi)^{2}}\left(\prod_{k=0}^{n-1} \int \frac{d^{3} \boldsymbol{k}_{k}}{(2 \pi)^{3}} \phi\left(q_{k}, \delta s_{k}\right)\right) J_{T}^{2}(k),
$$

where $\alpha_{s}$ is the coupling constant and $C_{R}=4 / 3(3)$ is the color averaged squared vertex for a gluon radiated off a quark (gluon) leg. The parton momentum distributions in the QGP are encoded as color and spatial averages of squared S-matrices in a classical background gauge field of tagged partons, and given by

$$
\phi(q, s)=2 \pi \delta\left(q^{0}\right) \int d^{2} \boldsymbol{x} e^{i \boldsymbol{q} \cdot \boldsymbol{x}} \exp \int_{0}^{s} d z \rho(z)[\sigma(\boldsymbol{x})-\sigma(\mathbf{0})],
$$

where $q$ is the momentum exchange of the gluon in a path of length $s, \rho(z)$ is the local number density of the medium at a depth $z$ and $\sigma(\boldsymbol{x})$ is the color averaged Fourier transform of the single elastic cross section for the leading order scattering amplitude $F_{e l}^{(1)}=-i 4 \pi \alpha_{s} t_{\alpha}^{A} t_{\alpha}^{R} /\left(\boldsymbol{q}^{2}+\mu_{d}^{2}(T)\right)$ with $t_{\alpha}^{A, R}$ the SU(3) matrices in the adjoint representation $A$ and the target parton representation $R$. The total squared emission current contains only the subset of gluon bremsstrahlung diagrams contributing to the intensity after a hard production vertex

$$
J_{T}^{2}(k)=\left|J^{n}+\sum_{l=1}^{n-1} J^{l}\right|^{2}-\left|J^{n}\right|^{2}, \quad J_{i}^{l}=\epsilon_{i j k} \frac{k_{j}^{l} p_{k}}{k_{\mu}^{l} p^{\mu}}\left(e^{i \varphi_{l+1}^{n}}-e^{i \varphi_{l}^{n}}\right), \quad J_{i}^{n}=\epsilon_{i j k} \frac{k_{j}^{n} p_{k}}{k_{\mu}^{n} p^{\mu}},
$$

where in the Coulomb gauge $J_{0}^{l}=J_{0}^{n}=0$ and $\epsilon_{i j k}$ is the Levi-Civita symbol. The hard particle 4-momentum $p=\left(p_{0}, \boldsymbol{p}\right)$ can be left fixed, in the soft gluon limit, in the particle initial direction after the production vertex. The phase difference $\varphi_{b}^{a}$ records the 4-current incoherence introduced between multiple interactions at $a$ and $b$ and modulates the non-Abelian LPM effect. It reads

$$
\varphi_{l}^{n}=\frac{1}{p_{0}} \sum_{i=l}^{n-1} \delta s_{i} k_{\mu}^{i} p^{\mu}, \quad \delta s_{i}=s_{i+1}-s_{i},
$$

where $s_{i}$ is the interaction point in the path and $p_{0}$ the hard particle energy.

To make connection with previous works, intensity in Eq. (1) resums at the amplitude level all the multiple scattering up to arbitrary and finite opacities, considering the full kinematics of the interactions, and admitting arbitrary forms of the perturbative cross sections and arbitrary profiles for static or expanding media [27, 28]. In the collinear limit $\omega \gg k_{\perp}$ well known results in the light cone are found [26]. In particular, path integrals matching the BDMPS-ASW [13, 17] result in the Fokker-Planck approximation for (2), or the more recent numerical implementation of the BDMPSASW expression for multiple hard scatterings [24]. On the other hand, the perturbative expansion reproduces the GLV series [8, 9]. In this collinear approximation, resummations for semi-infinite $[16,29]$ and finite media [30] have been known for a while with an approximate integration of the angular distribution of gluons. In the soft limit the distribution of multiple gluon emission is given by a classical Poisson process. Then the probability $P$ of a parton of initial energy $p_{\perp}$ of losing an energy $\epsilon$ is given by

$$
P\left(\epsilon, p_{t}\right)=\mathcal{N} \sum_{n=0}^{\infty} \frac{1}{n !} \delta\left(\epsilon-\sum_{i=1}^{n} \omega_{i}\right) \prod_{i=1}^{n} I\left(p_{t}-\sum_{j=1}^{i-1} \omega_{j}\right) .
$$


where $N$ is a normalization constant. The quenched cross section of observing an hadron of energy $p_{\perp}$ is then given by $[31,32]$

$$
\frac{d \sigma_{A A}^{h}\left(p_{t}\right)}{d y d p_{t}} \simeq T_{A A} \sum_{i} \int_{0}^{\infty} d \epsilon \frac{d \sigma_{p p}^{i, h}\left(p_{t}+\epsilon\right)}{d y d p_{t}} P_{i}\left(\epsilon, p_{t}+\epsilon\right) .
$$

where $i$ is the parton species, a light quark representative at high $p_{\perp}$ and a gluon at low $p_{\perp}$, and $T_{A A}$ the nuclear overlap function. In (6) the fragmentation function effects have been averaged at a typical fragmentation ratio. Finally the nuclear modification factor is defined as

$$
R_{A A}^{h} \equiv \frac{1}{\left\langle T_{A A}\right\rangle} \frac{d \sigma_{A A}^{h}\left(p_{t}\right)}{d y d p_{t}} / \frac{d \sigma_{p p}^{h}\left(p_{t}\right)}{d y d p_{t}} .
$$

To test the sensitivity of the nuclear modification factor (7) to the spectrum resummation, a QGP is implemented with Bjorken, longitudinal expansion with equilibrium temperature $T_{0}$ set by the energy density measurements and the scaling $\epsilon \tau_{0} \propto T_{0}^{3}$, where $\tau_{0} \propto 1 / T_{0}$ is the thermalization time [33]. The relation $\epsilon \tau_{0}=(8.85 \pm 0.44) \times\left(\sqrt{s_{n n}}\right)^{0.33 \pm 0.02}$, found for the most central classes between $\sqrt{s_{n n}}=27 \mathrm{GeV}-2.76 \mathrm{TeV}[34,35]$ is instead used when data has not been made available. Then, a reference temperature is set for the most central $\mathrm{PbPb}$ collisions at $\sqrt{s_{n n}}=2.76 \mathrm{TeV}$ to the value $T_{0}=470 \mathrm{MeV}$, following [36]. Once this temperature is set, all the parameters in the analysis are being fixed except for the QGP number density, which is the only unknown parameter given by the fit to the $R_{A A}^{h}$ data. The Debye screening mass $\mu_{d}(T)$, characterizing collisions with point-like scatterers in the QGP [37], and the strong coupling constant $\alpha_{s}(T)$, are given at leading order by $\mu_{d}^{2}(T)=4 \pi \alpha_{s}(T)\left(1+N_{f} / 6\right) T^{2}[38,39]$ and $\alpha_{s}(q)=1 /\left(b_{0} \ln \left(q^{2} / \Lambda^{2}\right)\right)$, respectively, with $b_{0}$ the 1-loop $\beta$-function coefficient for $N_{f}=2+1$ active flavors, and the momentum scales are set as $q=2 \pi T$ and $\Lambda_{s}=247 \mathrm{MeV}$ to match the world averaged data in [40]. This setup reproduces well the collected data up to the coldest system analyzed in this work, $\mathrm{Cu}-\mathrm{Cu}$ collisions at $\sqrt{s_{n n}}=200 \mathrm{GeV}$ at centrality $30 \%-40 \%$, and the color screening reproduces very well the pure gauge $\left(N_{f}=0\right)$ lattice computations, and falls slightly below of the $N_{f}=2$ flavor result in the staggered quark action [41]. The QGP lifetime is set with the relation $\tau_{f}=(0.87 \pm 0.01) \times\left(d N_{c h} / d \eta\right)^{1 / 3} \mathrm{fm} / \mathrm{c}$ found for the available data $[42,43]$ on Bose-Einstein pion correlations [44, 45], with kinetic freeze-out set to $T_{f}=120 \mathrm{MeV}$. For further details we refer to [46].

An analysis of existing data on jet quenching, including $\mathrm{Cu}-\mathrm{Cu}$ and $\mathrm{Au}-\mathrm{Au}$ data at $200 \mathrm{GeV}$ [47, 48], $\mathrm{Pb}-\mathrm{Pb}$ at $2.76 \mathrm{TeV}$ [49-51], $\mathrm{Pb}-\mathrm{Pb}$ at 5.02 $\mathrm{TeV}[52,53]$, and $\mathrm{Xe}-\mathrm{Xe}$ data at $5.44 \mathrm{TeV}$ [54] is made using the full order resummation as well as only the first term in the opacity expansion. For each case a fit is made to the nuclear modification factor $R_{A A}^{h}$ using $\rho\left(T_{0}\right)$ as the single unknown parameter. Results are collected in Fig.1. The found opacity using the full resummation of the spectrum roughly scales as $T_{0}^{3}$ with a slow decrease along the phase crossover. Unlikely, when only the first term in the perturbative expansion is kept, substantial deviations from the $T_{0}^{3}$ behavior are found as the temperature of the QGP decreases, energy to energy, centrality to centrality. A deviation factor of $K \simeq 1.22$, or of $K \simeq 1.29$ in terms of $\hat{q}$, the QGP transport parameter, is found when going from the most central Pb-Pb collisions at $\sqrt{s_{n n}}=2.76 \mathrm{TeV}$ in the LHC to the most central Au-Au collisions at $\sqrt{s_{n n}}=200 \mathrm{GeV}$ at RHIC. Results for $\hat{q}$ are shown in Fig.1. This factor is consistent with the value $K \simeq 1.3$ observed in the Jet Collaboration analysis [4] using the $N=1$ 
approximation. Larger factors $K \simeq 2$ have been found using the Gaussian approximation [5]. We thus conclude that for a description of the jet quenching data without temperature issues a full resummation of the medium induced gluon radiation is required. In addition, since the scalings seem to be consistent with the high temperature limit extrapolations, we compare our results for $\rho\left(T_{0}\right)$ with the lattice predictions for the entropy $s / 4 T^{3}$, pressure $p / T^{4}$ and energy density $\epsilon / 3 T^{4}$. These predictions are also shown in Fig. 1. Our result for the number density seems to agree rather well with the lattice predictions for the pressure, although under our current uncertainties - further discussed in [46] - it may be also consistent with either the entropy or energy density relations. This preliminary study may pave the path for more precise analyses, providing and additional handle to study the QCD Equation of State.
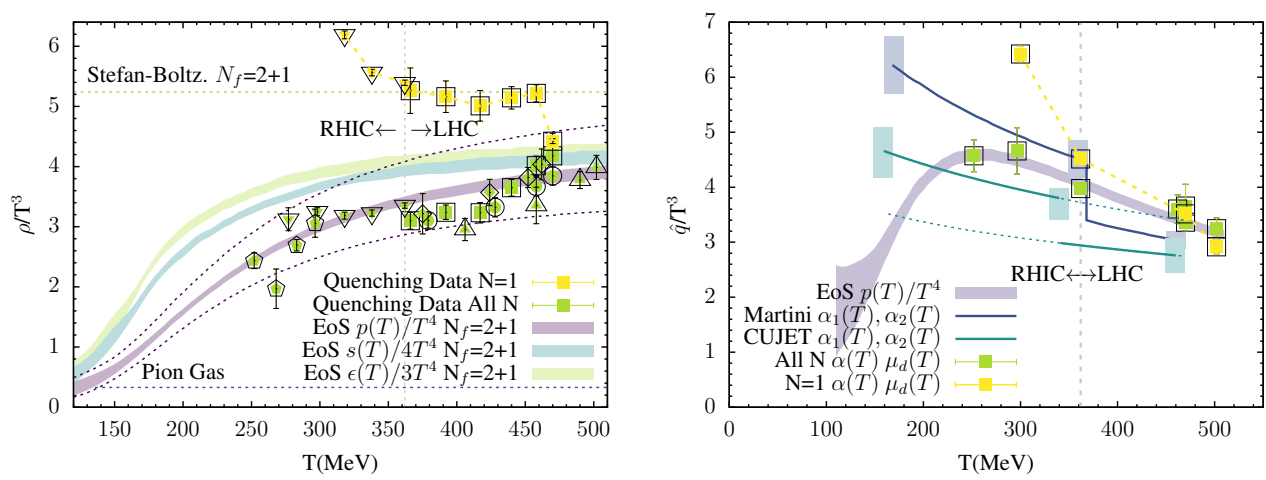

Figure 1: [Left] QGP density extracted from an All $N$ analysis (purple symbols ) and a first order $N=1$ analysis (green symbols) of the $R_{A A}^{h}$ collected data on collisions of $\mathrm{CuCu}$ (pentagons) and $\mathrm{AuAu}$ (down triangles) at $\sqrt{s_{n n}}=200 \mathrm{GeV}, P b P b$ at $\sqrt{s_{n n}}=2.76 \mathrm{TeV}$ (squares and circles), $P b P b$ at $\sqrt{s_{n n}}=5.02 \mathrm{TeV}$ (up triangles) and $\mathrm{XeXe}$ at $\sqrt{s_{n n}}=5.44 \mathrm{TeV}$ (diamonds) from PHENIX, ALICE and CMS Collaborations, compared to lattice results of the Equation of State by the Wuppertal collaboration [55]. [Right] QGP transport parameter $\hat{q}$ extracted from an all order analysis (green squares) or a fist order order analysis (yellow squares) of the $R_{A A}^{h}$ data. Also shown is the $\hat{q}$ assuming $\rho=p / T^{4}$ from lattice predictions of the QCD Equation of State [55] (green band), and the CUJET (blue) and MARTINI (purple) puzzles [4].

\section{Acknowledgments}

We thank the very valuable contributions of R.A. Vazquez and C.A. Salgado to this work. This work has been funded by Ministerio de Ciencia e Innovación of Spain under project FPA201783814-P; Unidad de Excelencia María de Maetzu under project MDM-2016-0692; ERC-2018ADG-835105 YoctoLHC; and Xunta de Galicia (Consellería de Educación) and FEDER. X.F. is supported by grant ED481B-2019-040 (Xunta de Galicia) and the Fulbright Visiting Scholar fellowship.

\section{References}

[1] Y. Akiba et al., Preprint, arXiv:1502.02730.

[2] Z. Citron et al., , CERN-LPCC-2018-07, arXiv:1812.06772. 
[3] A. Dainese et al., CERN-TH-2016-107, arXiv:1605.01389.

[4] K.M. Burke et al., Jet Collaboration, Phys. Rev. C 90014909 (2014).

[5] C. Andres, N. Armesto, M. Luzum, C.A. Salgado and P. Zurita, Eur. Phys. J. C 76 no.9 475 (2016).

[6] B. G. Zakharov, arXiv:2007.09772 [hep-ph].

[7] G. Y. Qin, J. Ruppert, C. Gale, S. Jeon, G. D. Moore and M. G. Mustafa, Phys. Rev. Lett. 100 (2008), 072301

[8] M. Gyulassy, P. Levai and I. Vitev, Phys. Rev. Lett. 855535 (2000).

[9] U.A. Wiedemann, Nucl. Phys. B 588303 (2000).

[10] L. Landau and I. Pomeranchuk, Dokl. Akad. Nauk Ser. Fiz. 92535 (1953).

[11] L. Landau, Dokl. Akad. Nauk Ser. Fiz. 92735 (1953).

[12] A.B. Migdal, Phys. Rev. 103181

[13] R. Baier, Yu.L. Dokshitzer, A.H. Mueller, S. Peigne and D. Schiff, Nucl. Phys. B 483291 (1997).

[14] R. Baier, Yu.L. Dokshitzer, A.H. Mueller, S. Peigne and D. Schiff, Nucl. Phys. B 484265 (1997).

[15] B. G. Zakharov, Phys. Atom. Nucl. 61 (1998), 838-854

[16] B.G. Zakharov, JETP Lett. 63952 (1996).

[17] C.A. Salgado and U.A. Wiedemann, Phys. Rev. D 68014008 (2003).

[18] N. Armesto, C.A. Salgado and U.A. Wiedemann, Phys. Rev. D 69114003 (2004).

[19] L. Apolinário, N. Armesto, J.G. Milhano and C.A. Salgado, , JHEP 1502119 (2015).

[20] M.D. Sievert and I. Vitev, Phys. Rev. D98 094010 (2018).

[21] X. Feal and R.A. Vazquez, Phys. Rev. D 98074029 (2018).

[22] Y. Mehtar-Tani, JHEP 1907 (2019) 057.

[23] M. D. Sievert, I. Vitev and B. Yoon, Phys. Lett. B 795 (2019) 502.

[24] C. Andres, L. Apolinário and F. Dominguez, arXiv:2002.01517 [hep-ph].

[25] J. Barata and Y. Mehtar-Tani, arXiv:2004.02323 [hep-ph].

[26] X. Feal, PhD Thesis, University of Santiago de Compostela. arXiv:1812.06903.

[27] R. Baier, Yu.L. Dokshitzer, A.H. Mueller and D. Schiff, Phys. Rev. C 581706 (1998). 
[28] C.A. Salgado and U.A. Wiedemann, Phys. Rev. Lett. 89092303 (2002).

[29] P. Arnold, G.D. Moore and L.G. Yaffe, JHEP 0206030 (2002).

[30] S. Caron-Huot and C. Gale, Phys. Rev. C 82 (2010), 064902 doi:10.1103/PhysRevC.82.064902

[31] R. Baier, Yu.L. Dokshitzer, A.H. Mueller and D. Schiff, JHEP 0109033 (2001).

[32] M.L. Miller, K. Reygers, S.J. Sanders and P. Steinberg, Ann. Rev. Nucl. Part. Sci. 57205 (2007).

[33] M. Strickland, Nucl. Phys. A 98292 (2019).

[34] A. Adare et al., PHENIX Collaboration, Phys. Rev. C 93024901 (2016).

[35] J. Adam et al., ALICE Collaboration, Phys. Rev. C. 94034903 (2016).

[36] P.F. Kolb and U. Heinz, Preprint, arXiv:0306084.

[37] F. D’Eramo, M. Lekaveckas, H. Liu and K. Rajagopal, , JHEP 1305 (2013) 031.1 (1956).

[38] E. Braaten and R.D. Pisarski, Nucl. Phys. B 337569 (1990).

[39] A.K. Rebhan, Phys. Rev. D 48 R3967 (1993).

[40] M. Tanabashi et al., Particle Data Group, Phys. Rev. D 98030001 (2018).

[41] O. Kaczmarek and F. Zantow, Phys. Rev. D 71114510 (2005).

[42] J. Adams et al., STAR Collaboration, Phys. Rev. C 71044906 (2005).

[43] J. Adam et al., ALICE Collaboration, Phys. Rev. C 93024905 (2015).

[44] A.N. Makhlin and Yu.M. Sinyukov, Z. Phys. C 3969 (1988).

[45] F. Retiere and M.A. Lisa, Phys. Rev. C 70044907 (2004).

[46] X. Feal, C.A. Salgado and R.A. Vazquez, , arXiv:1911.01309 .

[47] A. Adare et al. (PHENIX Collaboration), Phys. Rev. Lett. 101162301 (2008).

[48] A. Adare et al. (PHENIX Collaboration), Phys. Rev. Lett. 101232301 (2008).

[49] S. Chatrchyan et al., CMS Collaboration, Eur. Phys. J. C 721945 (2012).

[50] B. Abelev et al., ALICE Collaboration, Phys. Lett. B 72052 (2013).

[51] K. Aamodt et al., ALICE Collaboration, Phys. Rev. Lett. 106032301 (2011).

[52] V. Khachatryan et al., CMS Collaboration, JHEP 04039 (2017)

[53] J. Adam et al., ALICE Collaboration, Phys. Rev. Lett. 116222302 (2016).

[54] A.M. Sirunyan et al. (CMS Collaboration), JHEP 1810138 (2018).

[55] S. Borsanyi et al., Nature 539 no.7627 69 (2016). 